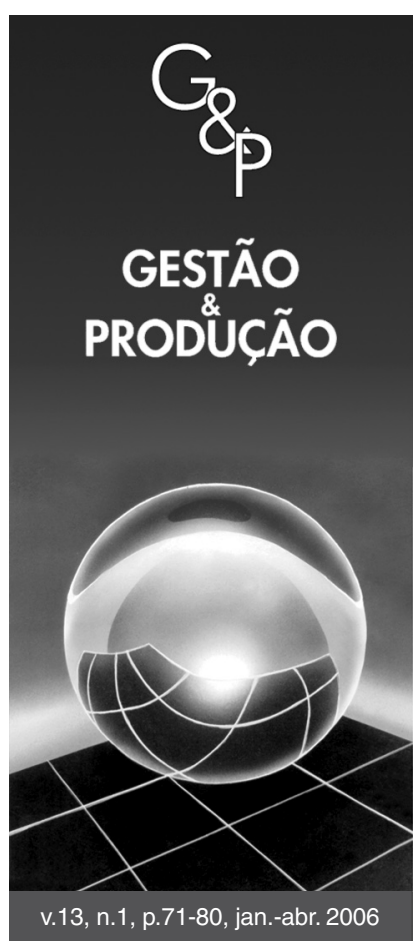

\section{EL EFECTO DEL TIPO DE PRODUCTO FABRICADOY DEL TAMAÑO DE LA EMPRESA EN LOS RESULTADOS PRODUCTIVOS DE LAS EMPRESAS DE PAVIMENTOS Y REVESTIMIENTOS CERÁMICOS DE LA COMUNIDAD VALENCIANA}

\author{
Juan Antonio Marín-Garcia \\ Grupo de Investigación en Recursos Humanos y Producción - i-GRHUP, \\ Departamento de Organización de Empresas, \\ Universidad Politécnica de Valencia - ETSIL, App. Correos 22012, 46071, Valencia, \\ e-mail: jamarin@omp.upv.es \\ www.upv.es/i.grup \\ Tomás Bonavía Martín \\ Grupo de Investigación en Recursos Humanos y Producción - i-GRHUP, \\ Area de Psicología Social, Facultad de Psicología, Universidad de Valencia, \\ Av. Blasco Ibáñez, 21, 46010, Valencia, España \\ e-mail:Tomas.Bonavia@uv.es \\ www.upv.es/i.grup \\ Recebido em 25/1/2005 \\ Aceito em 14/12/2005
}

\title{
Resumen
}

En este artículo, describimos los datos recogidos en las entrevistas realizadas a 101 directores de producción y en las visitas posteriores a sus plantas. En él, presentamos la importancia que tiene el tipo de producto fabricado y el número de trabajadores en plantilla, para explicar las diferencias que existen en los resultados productivos de las empresas. Algunos indicadores como el plazo de fabricación, la cantidad de inventario de producto terminado o la productividad por operario, son sensiblemente diferentes en las distintas agrupaciones de empresas. Sin embargo, la satisfacción percibida por el mando de producción apenas varía al agrupar las empresas por producto fabricado.

Palabras-clave: industrias azulejeras, Comunidad Valenciana, resultados productivos.

\section{Introducción}

El entorno de la mayoría de empresas industriales está caracterizado por un aumento de la rivalidad con las empresas competidoras, de la velocidad de los cambios y de la inestabilidad de la demanda. La mayoría de los mercados son maduros y sus clientes solicitan productos de calidad que se ajusten a sus necesidades específicas, una de ellas es la exigencia de entregas más frecuentes y rápidas (Marín y Delgado, 2000; Suzaki, 2000).

Las empresas que fabrican pavimentos y revestimientos cerámicos, especialmente en los principales países productores no son ajenas a estas circunstancias (Andrés Romano, 2001; Dalmau Porta et al., 1993; Rowley, 1996). Hasta hace pocos años, las principales variables de los procesos de producción eran el coste de la energía, el coste de la mano de obra y el acceso a una materia prima con pocos residuos (Ybarra et al., 1996). Sin embargo, desde principio de los años 90, los problemas tradicionales de producción se ven complicados por una nueva tendencia que obliga a repensar las estrategias de dirección de operaciones en el sector (Andrés Romano, 2001).

La demanda es cambiante, existe una competencia internacional cada vez más creciente en el sector y las empresas españolas intentan consolidarse en segmentos de mercado más rentables y exigentes. Todo ello ha originado que la gama de productos se amplíe, que se esté reorientando la producción hacia bienes de más calidad y diseño, que la empresas se hayan sensibilizado con la importancia de los controles de calidad y la necesidad de la flexibilización productiva (Tomás Carpi et al., 1996). Todo parece indicar que esta tendencia se verá incrementada en el futuro.

Los elementos clave para competir en este entorno, según las opiniones de los gerentes de las empresas consisten en mejorar el servicio al cliente y la calidad, 
reducir los plazos de entrega, mantener un precio razonable y ampliar la gama de producción (Tomás Carpi et al., 1996).

En definitiva, aparecen tres líneas prioritarias de actuación. Por un lado la mejora continúa de la calidad, que permitiría hacer frente a la necesidad de disminuir los defectos. Por otro, la mejora de la capacidad y flexibilidad de los procesos, que permitiría aumentar la rapidez del plazo de entrega y adaptar los productos a las características solicitadas por los clientes. Por último, la mejora de la productividad y eficiencia que permitiría reducir los costes de producción.

\section{Medición del rendimiento}

Si las empresas del sector quieren avanzar en alguna de las tres líneas de actuación comentadas en el apartado anterior, deberían ser capaces de medir los progresos en esa dirección, para lo cual necesitan un conjunto de indicadores útiles (Neely et al., 1997). Nuestra investigación se va a centrar exclusivamente en la eficiencia del departamento de producción, y más concretamente en la líneas de fabricación.

Un buen sistema de indicadores para el área de producción debe incluir indicadores no financieros lo suficientemente sencillos como para proporcionar feedd-back rápido de lo que sucede en la planta y permitir actuar para corregir las desviaciones no deseadas (Maskell, 1995). Los indicadores más comunes en las publicaciones científicas son: la productividad física (medida como piezas por operario), la calidad de los productos fabricados o la cantidad de inventario en la empresa (Cua et al., 2001; Giffi et al., 1990; Gunn, 1992; Lowe et al., 1997; Martínez Sánchez y Pérez Pérez, 2001; Maskell, 1995; White et al., 1999; Womack et al., 1992). Otros indicadores que suelen contemplarse son: la cantidad de entregas retrasadas, la reducción de producto devuelto por los clientes, los tiempos de preparación de las máquinas, plazo de fabricación (lead time) o el espacio necesario en el área de producción (Gunn, 1992; Lowe et al., 1997; Martínez Sánchez y Pérez Pérez, 2001; Maskell, 1995; Shah y Ward, 2003; White et al., 1999; Womack et al., 1992).

\section{Objetivos de la investigación}

Los directivos de producción pueden utilizar una serie de indicadores que les permitan ver la evolución de su sistema productivo en aquellas áreas relacionadas con las necesidades que hemos señalado como prioritarias al final del apartado de introducción. Teniendo en cuenta que la mayoría de las empresas del sector que vamos a estudiar son pequeñas, hemos procurado seleccionar indicadores muy sencillos (Gunasekaran et al., 2000) (Tabla 1).

Así, los indicadores seleccionados, relacionados con la necesidad de disminuir los defectos han sido el porcentaje de productos de primera calidad y la cantidad de productos devueltos por el cliente. También hemos incluido un indicador subjetivo como es el nivel de satisfacción del directivo de producción con la calidad de los productos que fabrican.

Otras de las necesidades importantes del sector son: atender el plazo de entrega solicitado por los clientes y ofrecer productos variados para responder a las diferentes necesidades de los clientes. Estas necesidades están relacionadas con el plazo de fabricación (tiempo desde que el cliente solicita un pedido hasta que se ha completado la fabricación del mismo), el porcentaje de pedidos entregados fuera de plazo, el tamaño del lote económico, el inventario de producto terminado almacenado y los indicadores subjetivos de satisfacción percibida por el mando de producción con la rapidez del plazo de entrega, la capacidad de ajuste con la demanda fluctuante y la adaptación del producto a las características solicitadas por el cliente.

Por último, la eficiencia del trabajo está relacionada con la productividad física de la planta (metros cuadrados producidos por operario) y la satisfacción con los costes de producción.

Tabla 1. Indicadores de resultados asociados a las principales necesidades del sector.

\begin{tabular}{lll}
\hline \multicolumn{1}{c}{ Disminuir defectos } & Plazos de entrega y variedad & \multicolumn{1}{c}{ Aumentar la eficiencia } \\
\hline - Primera calidad $(\%) ;$ & Plazo de fabricación; & • Productividad física; y \\
- Producto devuelto $(\%) ;$ y & - Inventario de producto terminado; & - Satisfacción con los costes de produc- \\
- Satisfacción con la calidad de los productos. & - Pedidos fuera de plazo $(\%) ;$ & ción. \\
& - Tamaño de lote económico; \\
& - Satisfacción con la rapidez en los plazos \\
& de entrega; \\
& - Satisfacción con el ajuste entre la canti- \\
& dad producida y la demanda; y \\
& - Satisfacción con la adaptación del pro- \\
& ducto a lo solicitado por el cliente. \\
\hline
\end{tabular}

Fuente: elaboración propia a partir de los datos de Fullerton y McWatters (2001), Maskell (1995) y Chiavenato (2000). 
La definción operativa de estos indicadores puede consultarse en el anexo.

Por otra parte, el valor que tomen los indicadores productivos, dependerá sin duda de lo bien o mal que se gestione el departamento de producción. Pero también es afectado por una serie de variables estructurales de la empresa. Probablemente, dos de las variables que más afectan a estos indicadores son el sector y el tamaño de la empresa (medido cómo número de empleados) (Cua et al., 2001; Kotha y Swamidass, 2000; Lowe et al., 1997).

La mayoría de las investigaciones han utilizado clasificaciones muy amplias. Así, por ejemplo, en la clasificación de sectores se suele dividir en industria, servicios y construcción. En la clasificación por tamaños, es habitual agrupar como empresas pequeñas a todas aquellas que tienen menos de 250 empleados. Sin embargo, creemos que podría ser interesante profundizar a un nivel mayor de detalle.

Por ello, un objetivo en esta investigación es analizar si los comportamientos demostrados en las clasificaciones amplias, se siguen manteniendo cuando analizamos uno de los subgrupos con más detalle. Concretamente, nos preguntamos si los conjuntos de empresas de un mismo sector que fabrican productos sensiblemente diferentes presentan indicadores productivos distintos, sólo por el hecho de fabricar determinado tipo de producto, independientemente de otras acciones de gestión de la producción. Del mismo modo, nos formularemos la misma pregunta cuando agrupamos las empresas pequeñas (menos de 250 trabajadores) en tres categorías: las más pequeñas del sector, tamaño medio y empresas pequeñas que casi son medianas empresas.

El sector industrial que hemos seleccionado, el de las empresas fabricantes de pavimentos y revestimientos cerámicos, tiene varias ventajas. Por un lado, se trata de uno de los principales sectores industriales de España, con empresas líderes a nivel mundial, lo que permite disponer de una población de estudio bastante amplia. Además, el $90 \%$ de las empresas de este sector tienen menos de 200 trabajadores (ASCER, 2003). Por otro lado, la homogeneidad de productos y procesos permite aislar unas pocas variables (tamaño de las empresas y tipo de producto fabricado), manteniendo relativamente constante el resto de variables (tipo de proceso, distribución en planta, nivel tecnológico, complejidad de productos, tiempos de fabricación). Además, la concentración geográfica en una zona delimitada permite que muchas variables de contexto como formación de la fuerza laboral, estructuras salariales o inversiones públicas sean muy similares para todas las empresas. Por último, no debemos olvidar que disponer de la población en un área geográfica próxima permite la recogida de los datos directamente de las empresas, sin elevar excesivamente los costes de la investigación.
A pesar de la importancia del sector de pavimentos y revestimientos cerámicos dentro de los sectores industriales españoles, hemos encontrado muy pocas publicaciones científicas españolas o estudios sectoriales con datos de indicadores productivos de las empresas. En ellas sólo se presentan datos acerca de la productividad por empleado, cuyos valores se situaban en el año 2001 en $2.265 \mathrm{~m}^{2}$ mensuales/operario (una evolución muy importante si los comparamos con los 645 de 1980 o los 1.381 de 1991) (ASCER, 2003; Dalmau Porta et al., 1993; Tomás Carpi et al., 1996). A nivel internacional, no hemos encontrado ningún artículo científico que refleje las variables contenidas en nuestra investigación y queremos contribuir a llenar ese hueco.

Resumiendo, además de presentar los niveles de los indicadores productivos de un conjunto de empresas punteras a nivel internacional, queremos demostrar las siguientes hipótesis:

- Hipótesis 1: dentro del sector de pavimentos y revestimientos cerámicos, los resultados de producción de las empresas varían en función del tipo de producto fabricado mayoritariamente por la empresa; y

- Hipótesis 2: los resultados de producción de las empresas, agrupadas por el número de empleados, son significativamente diferentes.

\section{Trabajo de campo}

Los datos que han servido de base para la elaboración de este informe se recogieron durante los meses de julio a septiembre de 2001. Previamente habíamos realizado una presentación del proyecto ante el comité de producción de ASCER (asociación española de fabricantes de azulejos y pavimentos cerámicos), que nos dio el visto bueno y nos prestó su apoyo, tanto para facilitar el contacto con sus asociado como para revisar y mejorar el borrador del cuestionario. Una vez completado el pase piloto en tres empresas del sector, contactamos telefónicamente con las empresas participantes para concertar una cita con el director de producción. En la fecha concertada, se realizó una entrevista en la que se completó un cuestionario y, al finalizar, visitamos las instalaciones de fabricación para rellenar algunos de los datos mediante observación directa del entrevistador. La duración promedio de estas dos actividades fue de una hora y media por planta.

Para la investigación, seleccionamos las 157 empresas asociadas a ASCER que fabricaban pavimentos o revestimientos y tenían su planta situada en la Comunidad Valenciana. 101 directores de producción (64\%) contestaron nuestras entrevistas. Para los análisis hemos agrupado a las empresas en función del tipo de producto fabricado y del tamaño de las mismas (Tabla 2). Por tipo de producto, la mayoría de las empresas participantes (76\%), fabrica- 
ban productos de monococción prensados. Este grupo lo dividimos en varias categorías: pavimento de gres porcelánico (14 empresas), fabricación indistinta de pavimento o revestimiento mediante monococción (27 empresas), fabricación sólo de pavimento de gres (23 empresas), fabricación sólo de revestimientos mediante monococción (12 empresas). El siguiente grupo (14\%) fueron las empresas de tercer fuego que fabricaban piezas especiales. Por último, un $12 \%$ de las empresas fabricaban productos extrudidos o revestimientos mediante bicocción.

Para agrupar las empresas por tamaño, hemos utilizado como variable identificadora el número de empleados de la empresa. Teniendo en cuenta la clasificación habitual en el mundo académico, la mayoría de las empresas estudiadas se considerarían pequeñas (menos de 250 trabajadores). Para adaptarnos a la realidad del tejido empresarial español y, más concretamente, del sector de pavimentos y revestimientos cerámicos, en la clasificación empleada en este artículo, hemos considerado que el valor de referencia para las empresas de tamaño medio sea de 100 trabajadores, esta categoría agrupa a 51 empresas (el $50 \%$ de la muestra) que tienen entre $50 \mathrm{y}$ 150 trabajadores empleados. Las 20 empresas con menos de 50 trabajadores se han considerado como las más pequeñas de la muestra y las empresas con más de 150 empleados (28 casos) se han considerado como las empresas más grandes de la muestra (Tabla 2). Esta clasificación es congruente con las utilizadas en otros trabajos (AS-
CER, 2003; ASTER, 1997; Lee y Palmer, 1999; Tomás Carpi et al., 1996).

Otras características de las empresas que hemos incorporado medido son el tamaño de los productos fabricados, el precio promedio de venta de los productos y el porcentaje de las ventas que se dedica a la exportación o que se vende directamente a las constructoras. Consideramos que estos datos pueden ayudar a contextualizar la situación de las empresas participantes en la investigación. En la Tabla 3 resumimos el promedio de toda la muestra y los promedios en función del número de trabajadores en plantilla.

Los productos de extrusión y piezas especiales son significativamente más pequeños $(\alpha<0,05$, prueba ANOVA con post-test DMS). El 97\% de los productos de estas empresas tienen todas sus dimensiones inferiores a los $40 \mathrm{~cm}$. En las empresas que sólo hacen revestimientos, bien sea por monococción o por bicocción, también son mayoritarios este tipo de productos (el $85 \%$ y el $70 \%$, respectivamente). El resto de empresas fabrican un $62 \%$ de sus productos con formato pequeño y en las empresas de gres porcelánico esta cifra se reduce hasta un 55\%.

El precio de venta promedio de los productos es significativamente mayor en las empresas que fabrican productos extrudidos, piezas especiales y gres porcelánico, con precios de venta por encima de los 6 euros por metro cuadrado. Por el contrario, los productos de revestimiento de monoccoción o bicocción son los más baratos (en

Tabla 2. Distribución de las empresas de la muestra por tipo de producto y número de empleados.

\begin{tabular}{|c|c|c|c|c|}
\hline & $\begin{array}{l}\text { Las más pequeñas } \\
<50 \text { empleados }\end{array}$ & $\begin{array}{c}\text { Intermedias } \\
\text { 50-150 empleados }\end{array}$ & $\begin{array}{l}\text { Las más grandes } \\
>150 \text { empleados }\end{array}$ & Total \\
\hline Extrusion & 3 & 2 & 0 & 5 \\
\hline Piezas especiales & 2 & 9 & 3 & 14 \\
\hline Pavimento de gres porcelánico & 1 & 1 & 12 & 14 \\
\hline Monococción pavimento y revestimiento & 1 & 15 & 9 & 25 \\
\hline Sólo pavimento monococción gres & 4 & 16 & 3 & 23 \\
\hline Sólo revestimientos monococción & 7 & 5 & 0 & 12 \\
\hline Revestimientos bicocción & 2 & 3 & 1 & 6 \\
\hline Total & 20 & 51 & 28 & 99 \\
\hline
\end{tabular}

Nota: el número de empleados de dos de las empresas, que fabricaban pavimentos y revestimientos por monococción, no fue facilitado, por lo que no aparecen en la tabla.

Tabla 3. Características generales de las empresas en función de su tamaño.

\begin{tabular}{lcccc}
\hline Plantilla & Exportación (\%) & Constructoras (\%) & $\begin{array}{c}\text { Formato pequeño } \\
(<\mathbf{4 0} \mathbf{~ c m}) \mathbf{( \% )}\end{array}$ & Precio promedio del $\mathbf{~ m}^{2}$ \\
\hline$<50$ & $43 \% *$ & $3,4 \%$ & $83 \%$ & $4,52 € *$ \\
$50-150$ & $51 \%$ & $5,5 \%$ & $71 \%$ & $5,01 €$ \\
$>150$ & $57 \%$ & $8,6 \%$ & $56 \% *$ & $5,50 €$ \\
Promedio & $51 \%$ & $5,7 \%$ & $71 \%$ & $5,19 €$ \\
\hline
\end{tabular}

*diferencia significativa $(\alpha<0,05)$ con los valores de los otros grupos, utilizando un ANOVA de un factor con post test DMS. 
promedio $3,8 €$ y $4,2 €$ por metro cuadrado, respectivamente).

Las empresas que fabrican productos extrudidos y piezas especiales, exportan un $30 \%$ y un $35 \%$ de sus productos, respectivamente. Estas cifras son notablemente inferiores $(\alpha<0,05$, prueba ANOVA con post-test DMS) a las de las empresas que fabrican solo revestimiento de monococción (42\%) o sólo pavimentos de gres $(55 \%)$ y, por supuesto, a la de las empresas de bicocción, que exportan en promedio el $63 \%$ de su producción.

El porcentaje de ventas a constructoras es casi insignificante en bicocción $(0,5 \%)$ o piezas especiales $(2 \%)$, mientras que el resto de empresas venden casi un $6 \%$ de su producción directamente a las constructoras, salvo las empresas que fabrican monococción de gres y porosa simultáneamente, que llegan hasta un $10 \%$.

Casi todas las empresas observadas presentan una distribución en planta muy similar. Los productos se fabrican en líneas en paralelo que empiezan con las prensas. A continuación de ellas se colocan las instalaciones para los acabados superficiales. Antes de entrar en los hornos se suelen disponer de un almacenamiento intermedio que permite regular la entrada del producto a los hornos. A la salida de los hornos se coloca una sección de clasificación y paletizado. En general, las empresas que realizan productos de gres porcelánico son las más grandes y tecnificadas. Coincide también que suelen ser las plantas más modernas y de construcción más reciente.

\section{Resultados del área de producción}

Para probar las hipótesis planteadas en nuestra investigación, hemos resumido los valores de las variables de re- sultados productivos, agrupándolos por tipo de producto fabricado y por número de empleados de las empresas.

En primer lugar analizaremos las variables cuyos valores se obtienen con datos objetivos y posteriormente analizaremos las variables cuyos valores proceden de percepciones subjetivas de los directores de producción.

En la Tabla 4, podemos observar que los niveles de producto de primera calidad son muy elevados y apenas hay diferencias notables entre los grupos de empresas. Una prueba adicional del elevado índice de calidad de la producción del sector es el porcentaje tan pequeño de producto devuelto por los clientes.

Las entregas fuera de plazo rondan el 9\% del total suministrado, siendo un poco menores en extrusión y bicocción, donde se cumplen mejor los plazos estipulados. Respecto al plazo medio de fabricación, es sensiblemente menor en las empresas de extrusión y de gres porcelánico donde transcurren unas dos semanas desde que se solicita el pedido hasta que se completa su fabricación. En el resto de empresas, esta cifra es superior a las tres semanas.

En promedio, las empresas del sector almacenan como producto terminado una cantidad de metros cuadrados equivalente a dos meses de producción. Sin embargo, aparecen diferencias significativas en estos valores según sea el tipo de producto que se fabrique. Así, por ejemplo, las empresas de revestimientos por monococción porosa y las de piezas especiales almacenan menos que el resto, mientras las empresas de bicocción y las de gres porcelánico son las que más inventario de producto terminado mantienen.

Por último, la productividad medida como $\mathrm{m}^{2}$ de fabricación mensual dividida por la cantidad de trabajadores de la empresa es mucho menor en las empresas de gres

Tabla 4. Promedio de los indicadores de producción por tipo de producto fabricado.

\begin{tabular}{|c|c|c|c|c|c|c|c|c|}
\hline & $\mathbf{E x t}$ & PzEsp & GresPorce & Monoc & SoloPavGr & SoloRevMon & RevBicc & $\begin{array}{c}\text { Promedio } \\
\text { sector }\end{array}$ \\
\hline Primera calidad (\%) & $92 \%$ & $89 \%$ & $90 \%$ & $89 \%$ & $89 \%$ & $89 \%$ & $88 \%$ & $89 \%$ \\
\hline $\begin{array}{l}\text { Producto no aceptado } \\
\text { por el cliente }(\%)\end{array}$ & $1 \%$ & $3 \% *$ & $1 \%$ & $1 \%$ & $1 \%$ & $1 \%$ & $0 \%$ & $1 \%$ \\
\hline Entregas fuera plazo (\%) & $2 \%$ & $10 \%$ & $6 \%$ & $10 \%$ & $11 \%$ & $6 \%$ & $3 \%$ & $9 \%$ \\
\hline $\begin{array}{l}\text { Días transcurridos } \\
\text { entre pedido y fin } \\
\text { de fabricación }\end{array}$ & $15,40 *$ & 22,69 & $15,31 *$ & 27,87 & 21,35 & 20,58 & 21,17 & 21,86 \\
\hline $\begin{array}{l}\text { Dias inventario } \\
\text { producto terminado }\end{array}$ & 53,80 & $40,85^{*}$ & 88,08 & 60,26 & 71,26 & $25,67 *$ & $109,50 *$ & 62,47 \\
\hline $\mathrm{M}^{2}$ Lote económico & 3.250 & 2.250 & $5.750 *$ & 3.703 & 4.011 & 2.221 & 1.716 & 3.681 \\
\hline $\begin{array}{l}\text { M}^{2} \text { Producción } \\
\text { mensual por operario }\end{array}$ & No disponible & No disponible & $2.611^{*}$ & 2.934 & 3.458 & 3.909 & 2.725 & 3.170 \\
\hline
\end{tabular}

Ext: extrusión; PzEsp: Piezas especiales: tercer fuego; GresPorce: pavimento de gres porcelánico; Monoc: monococción pavimentos y revestimientos; SoloPavGr: solo pavimento monococción gres; SoloRevMon: sólo revestimientos monococción porosa; y RevBicc: revestimientos bicocción.

*diferencia significativas $(\alpha<0,05)$ con los valores de los otros grupos, utilizando un ANOVA de un factor con post test DMS. 
porcelánico y las de bicocción que en las empresas que sólo fabrican revestimientos o pavimentos por monococción. Probablemente esto sea debido tanto a las características y complejidad de los procesos como al hecho de que las empresas de gres porcelánico sean las de mayor tamaño y que nuestra variable de productividad esté inversamente relacionada con el número de empleados, como veremos más adelante. No disponemos de datos de productividad de las empresas de extrusión ni de piezas especiales por no ser aplicable para sus procesos el indicador que hemos escogido en este estudio.

El tamaño de las empresas apenas afecta a algunos de los indicadores de producción. No hemos apreciado diferencias entre las empresas pequeñas, medianas y grandes, en cuanto a porcentaje de producto de primera calidad, porcentaje de entregas fuera de plazo, cantidad de producto devuelto o tamaño de lote económico.

Sin embargo, el plazo de fabricación es 10 días mayor en las empresas más grandes que en las más pequeñas $(\alpha<0,05$, prueba ANOVA con post-test DMS), mientras que la cantidad de producto terminado en almacenes es mucho menor $(\alpha<0,05$, prueba ANOVA con post-test DMS) en las pequeñas (26 días de fabricación) que en las medianas (68 días) o las grandes (73 días). Respecto a la productividad por operario, es mayor en pequeñas (3.893 $\mathrm{m}^{2}$ /operario) y medianas (3.348 $\mathrm{m}^{2} /$ operario) que en las más grandes ( $2.214 \mathrm{~m}^{2} /$ operario $)(\alpha<0,05$, prueba ANOVA con post-test DMS).

El otro conjunto de indicadores observado lo constituyen la satisfacción percibida por los mandos de producción sobre distintos aspectos relacionados con su departamento. El aspecto mejor valorado es la calidad de los productos fabricados, sin apenas diferencia entre los diferentes grupos de empresas. A continuación, los direc- tores de producción se sienten bastante satisfechos con la adaptación del producto a las características solicitadas por el cliente. Podemos observar que en las empresas de piezas especiales esta satisfacción es mayor que en el resto de grupos, salvo el de bicocción. La satisfacción es moderada en la rapidez de plazos de entrega y la capacidad de ajustar la producción a la demanda. Mientras que la satisfacción con los costes de producción es menor, con ligeras diferencias entre los grupos (Tabla 5).

Hemos encontrado algunas diferencias significativas $(\alpha<0.05$, prueba ANOVA con post-test DMS) en la satisfacción percibida al analizarla en función del tamaño de las empresas. Por un lado, las empresas pequeñas están más satisfechas que las más grandes en la rapidez de los plazos de entrega. Por otro lado, las empresas medianas se muestran más satisfechas que las grandes respecto a la capacidad de ajuste de la producción a la demanda. Por último, las empresas más pequeñas muestran más satisfacción con los costes de producción que las empresas medianas o más grandes.

\section{Análisis de resultados}

En cuanto a las dos hipótesis planteadas, los resultados obtenidos permiten validarlas parcialmente (ver Tabla 6).

Así, para la hipótesis 1, hemos comprobado que en los indicadores objetivos de "plazo de fabricación", "cantidad de producto terminado en almacenes", " $\mathrm{m}^{2}$ de producción por operario", " $\mathrm{m}^{2}$ de lote económico" y "\% producto no aceptado por el cliente" existen diferencias significativas en los valores obtenidos por las empresas que fabrican un determinado tipo de producto. Normalmente, se trata de uno o dos conjuntos de empresas los que se diferencias claramente del resto y no siempre son

Tabla 5. Satisfacción percibida por los directores de producción.

\begin{tabular}{lcccccccc}
\hline & Ext & PzEsp & GresPorce & Monoc & SoloPavGr & SoloRevMon & $\begin{array}{c}\text { RevBicc } \\
\text { Promedio } \\
\text { sector }\end{array}$ \\
\hline $\begin{array}{l}\text { Calidad de los productos } \\
\text { fabricados }\end{array}$ & 4,20 & 4,21 & 4,14 & 4,15 & 4,00 & 4,08 & 4,33 & 4,13 \\
$\begin{array}{l}\text { Adaptación producto a lo } \\
\text { solicitado por el cliente }\end{array}$ & 4,00 & $4,43^{*}$ & 3,93 & 4,07 & 3,98 & 4,08 & 4,20 & 4,09 \\
$\begin{array}{l}\text { Rapidez de los plazos de } \\
\text { entrega }\end{array}$ & 3,60 & 3,57 & 3,86 & 3,52 & 3,43 & 3,67 & 4,00 & 3,60 \\
$\begin{array}{l}\text { Capacidad de ajustar la } \\
\text { producción a la demanda }\end{array}$ & 4,00 & 3,71 & 3,36 & 3,70 & 3,43 & 3,33 & 4,00 & 3,58 \\
\begin{tabular}{l} 
Costes de producción \\
\hline
\end{tabular} & 3,40 & 3,43 & 3,00 & 3,11 & 2,85 & 3,25 & 3,50 & 3,13 \\
\hline
\end{tabular}

Variables medidas de 1 (poco satisfecho) a 5 (muy satisfecho);

Ext: extrusión; PzEsp: Piezas especiales, tercer fuego; GresPorce: pavimento de gres porcelánico; Monoc: monococción pavimentos y revestimientos; SoloPavGr: solo pavimento monococción gres; SoloRevMon: sólo revestimientos monococción porosa; y RevBicc: revestimientos bicocción.

*diferencia significativas $(\alpha<0,05)$ con los valores de los otros grupos, utilizando un ANOVA de un factor con post test DMS. 
Tabla 6. Comprobación de hipótesis.

\begin{tabular}{|c|c|c|c|}
\hline Tipo de indicador & Indicador & $\begin{array}{c}\text { Diferencias significativas } \\
\text { Hipotesis } 1 \\
\text { (tipo de producto fabricado) }\end{array}$ & $\begin{array}{c}\text { Diferencias significativas } \\
\text { Hipótesis } 2 \\
\text { (tamaño de las empresas) }\end{array}$ \\
\hline \multirow[t]{7}{*}{ Objetivo } & Primera calidad $(\%)$. & Ninguna. & Ninguna. \\
\hline & $\begin{array}{l}\text { Producto no aceptado por } \\
\text { el cliente }(\%) \text {. }\end{array}$ & Piezas especiales mayor que el resto. & Ninguna. \\
\hline & Entregas fuera plazo $(\%)$ & Ninguna. & Ninguna. \\
\hline & $\begin{array}{l}\text { Días transcurridos entre } \\
\text { pedido y fin de fabricación. }\end{array}$ & $\begin{array}{l}\text { Extrusión y Gres porcelánico } \\
\text { menor que el resto. }\end{array}$ & $\begin{array}{l}\text { Mayor en las más grandes que en las más } \\
\text { pequeñas. }\end{array}$ \\
\hline & $\begin{array}{l}\text { Días inventario producto } \\
\text { terminado. }\end{array}$ & $\begin{array}{l}\text { Piezas especiales y revestimientos } \\
\text { monococción porosa menos que el resto } \\
\text { Revestimientos bicocción mayor que el } \\
\text { resto. }\end{array}$ & $\begin{array}{l}\text { Menor en las más pequeñas que en las } \\
\text { medianas o más grandes. }\end{array}$ \\
\hline & M² Lote económico. & Gres porcelánico mayor que el resto. & Ninguna. \\
\hline & $\begin{array}{l}\mathrm{M}^{2} \text { Producción mensual } \\
\text { por operario. }\end{array}$ & $\begin{array}{l}\text { Gres porcelánico menor que el resto } \\
\text { (no se dispone del dato de piezas } \\
\text { especiales y extrusión). }\end{array}$ & $\begin{array}{l}\text { Mayor en las más pequeñas y medianas } \\
\text { que en las más grandes. }\end{array}$ \\
\hline \multirow[t]{5}{*}{ Subjetivo } & $\begin{array}{l}\text { Calidad de los productos } \\
\text { fabricados. }\end{array}$ & Ninguna. & Ninguna. \\
\hline & $\begin{array}{l}\text { Adaptación producto a lo } \\
\text { solicitado por el cliente. }\end{array}$ & $\begin{array}{l}\text { Mayor en piezas especiales } \\
\text { que en el resto. }\end{array}$ & Ninguna. \\
\hline & $\begin{array}{l}\text { Rapidez de los plazos de } \\
\text { entrega. }\end{array}$ & Ninguna. & $\begin{array}{l}\text { Mayor en las más pequeñas que en las } \\
\text { más grandes. }\end{array}$ \\
\hline & $\begin{array}{l}\text { Capacidad de ajustar la } \\
\text { producción a la demanda. }\end{array}$ & Ninguna. & $\begin{array}{l}\text { Mayor en las medianas que en las más } \\
\text { grandes. }\end{array}$ \\
\hline & Costes de producción. & Ninguna. & $\begin{array}{l}\text { Mayor en las más pequeñas que en las } \\
\text { medianas o las más grandes. }\end{array}$ \\
\hline
\end{tabular}

los mismos en todos los indicadores. Por otra parte, no hemos encontrado diferencias en el "\% de primera calidad" ni las "entregas fuera de plazo".

Prácticamente ninguno de los indicadores subjetivos se veía afectado por el tipo de producto fabricado por las empresas.

Por lo tanto, consideramos que para las investigaciones que recojan indicadores objetivos de las líneas de producción, se debe tener en cuenta, no solo el sector, sino también el tipo de producto fabricado por las empresas para poder explicar las diferencias en sus valores. Sin embargo, con los datos de nuestra investigación no podemos asegurar que sea necesario tanto nivel de detalle para los indicadores subjetivos, pudiendo ser suficiente con una clasificación por sectores.

Para la hipótesis 2, si observamos los indicadores objetivos, sólo el "plazo de fabricación", la "cantidad de producto terminado en almacenes" y la "productividad por operario" presentan diferencias significativas para las agrupaciones por tamaño de las empresas.

La "satisfacción percibida por los directivos de producción con los plazos de entrega" es mayor en las empresas pequeñas que en las mas grandes. Probablemente esto sea debido a que su plazo de fabricación es menor ya que las empresas pequeñas tienen, en promedio, me- nos producto terminado en almacenes que las grandes. La combinación de estos dos indicadores objetivos también puede explicar por qué los directivos de las empresas medianas se muestran más satisfechos que los de las empresas grandes con el "ajuste de la producción a la demanda". Por último, las empresas pequeñas parecen más satisfechas con los costes, y esto concuerda con el dato de una mayor productividad por operario. El resto de indicadores subjetivos no presenta diferencias significativas al agrupar las empresas por tamaño.

En definitiva, con los indicadores objetivos podemos encontrar diferencias significativas entre las agrupaciones de empresas realizadas por el tipo de producto fabricado, quedando las diferencias poco resaltadas con los indicadores subjetivos. Sin embargo, cuando observamos las diferencias debidas al tamaño de la empresa, se llegan a visiones análogas tanto con los indicadores objetivos como con los subjetivos.

Una de las explicaciones probables es que los indicadores subjetivos disponen sólo de 5 valores posibles (muy poco, poco, regular, bastante, mucho) y la mayoría de respuestas se concentran en la parte alta de la escala, es decir, existe menos variabilidad de las respuestas en los indicadores subjetivos que en las de los indicadores objetivos. Si creamos muchos grupos al realizar el análi- 
sis, cosa que ocurre con los 8 grupos de la clasificación por tipo de productos, la cantidad de sujetos en cada grupo disminuye y con ello, también disminuye la posibilidad de que un determinado efecto sea considerado con significación estadística. Este problema no es tan manifiesto al usar las agrupaciones por tamaño donde sólo se crean 3 categorías.

\section{Conclusiones y consideraciones finales}

Casi todas las empresas observadas presentan una distribución en planta muy similar, con unos procesos muy parecidos y con escasas diferencias en cuanto a la tecnología empleada. Además, la percepción de los aspectos claves para competir son también muy semejantes: mejorar el servicio al cliente y la calidad, reducir los plazos de entrega, mantener un precio razonable y ampliar la gama de producción.

El niveles de calidad de los productos es muy elevado ( $89 \%$ de producto de primera calidad y menos del $1 \%$ de producto devuelto por los clientes). Apenas hay diferencias notables entre las empresas que fabrican diferentes tipos de productos, así como tampoco hemos observado diferencias debidas al tamaño de las empresas. En las entregas fuera de plazo tampoco hemos observado comportamientos diferentes entre las empresas de la muestra.

El plazo medio de fabricación ronda las tres semanas y es sensiblemente menor en las empresas de extrusión y de gres porcelánico. Las empresas más grandes presentan plazos de fabricación mayores. El tipo de producto fabricado también afecta a otras variables como los niveles de inventario, tamaño del lote económico y productividad de las empresas.

Del mismo modo, el plazo de fabricación, la cantidad de producto terminado en almacenes y la productividad presentan valores promedios diferentes cuando comparamos las empresas agrupadas por tamaño.

Debido a estas diferencias, creemos que nuestra investigación aporta datos interesantes para el "benchmarking" de las empresas ya que en lugar de presentar los promedios sectoriales de determinados indicadores productivos, los agrupamos en categorías de empresas más homogéneas (empresas que fabrican un producto similar o que tienen un tamaño parecido). De este modo proporcionamos a las empresas del sector la oportunidad de descubrir posibles aspectos productivos a mejorar.

Con respecto a la satisfacción percibida por los mandos de producción sobre distintos aspectos relacionados con su departamento, solo hemos podido observar que en las empresas de piezas especiales la satisfacción con la adaptación del producto a las solicitudes de los clientes es mayor que en el resto de grupos. En el resto de variables, no se aprecian diferencias por tipo de producto fabricado.

Sin embargo, la satisfacción percibida con lo plazos de entrega, el ajuste a la demanda y los costes de producción tiende a ser menor en las empresas grandes. Este resultado nos induce a pensar que precisamente serán las empresas grandes las más interesadas en realizar cambios y mejoras en sus sistemas productivos. No sólo porque dispongan de mayores recursos, sino también porque consideran estos cambios más necesarios.

\subsection{Limitaciones}

Una de las limitaciones de la investigación es que las conclusiones no se puede generalizar a otros sectores, ya que todas las empresas de la muestra pertenecen al sector de fabricantes de pavimentos y revestimientos cerámicos.

Otra limitación es que la tasa de respuesta es del 64\%. Aunque se trata de una cifra muy elevada para investigaciones de este tipo, debemos ser prudentes al extrapolar los resultados. Sobre todo los de aquellas agrupaciones de empresas con pocos casos en la muestra.

Por último, debemos interpretar con cautela las conclusiones derivadas de las percepciones de los mandos de producción. Por tratarse de datos subjetivos, es probable que niveles altos de satisfacción no se correspondan con una mayor eficiencia productiva, sino con una menor exigencia o expectativas del mando.

\subsection{Investigación futura}

Pretendemos continuar investigando el comportamiento de la empresas del sector, para identificar si determinadas acciones de gestión de producción y de gestión de recursos humanos, permiten explicar las diferencias en los resultados productivos. Concretamente nos interesaría estudiar las actividades relacionadas con la implantación de aspectos asociados al modelo de producción ajustada y al aumento de la participación de los trabajadores. En este sentido, las conclusiones de este artículo son muy útiles pues nos indican que debemos tener en cuenta el tipo de producto fabricado y el tamaño de la empresa como variables de control de la investigación futura.

\section{Agradecimientos}

Para la realización de este trabajo contamos con el apoyo de la Consellería de Cultura y Educación de la Generalitat Valenciana (GV00-013-7). También agradecemos al Área de Coordinación de Lenguas Extranjeras de la Universidad Politécnica de Valencia, su ayuda en la traducción del resumen y a ASCER el apoyo brindado a lo largo del trabajo. 


\section{Anexo}

La definición operativa de las variables dependientes utilizadas para evaluar la eficiencia del proceso productivo, fueron las siguientes:

- Primera calidad (\%): porcentaje de piezas correctas, respecto al total de piezas que entran en un proceso;

- Producto devuelto (\%): porcentaje de producto devuelto por el cliente, respecto del total de piezas suministradas;

- Plazo de fabricación: tiempo promedio que transcurre entre que se recibe un pedido en el departamento de producción y se termina su fabricación;
- Inventario de producto terminado: $\mathrm{m}^{2}$ de producto terminado en los almacenes de la empresa;

- Pedidos fuera de plazo (\%): porcentaje de pedidos suministrados fuera del plazo pactado con el cliente;

- Tamaño de lote económico: cantidad mínima de $\mathrm{m}^{2}$ que se fabrican en un lote para que sea rentable;

- Productividad física.: cantidad de $\mathrm{m}^{2}$ producidos por operario en un mes; $y$

- Todas las variables de percepción de satisfacción del mando se midieron en una escala de 1 (poco satisfecho) a 5 (muy satisfecho).
ANDRÉS ROMANO, C. Problemática de programación de producción en la empresa cerámica, $\mathrm{PhD}$ thesis, 2001, Business Administration. Academic Department: Organización de empresas. Universidad Politécnica de Valencia, 2001.

ASCER - EL SECTOR ESPAÑOL DE FABRICANTES DE BALDOSAS CERAMICAS. Asociación de Fabricantes de Azulejos, pavimentos y Baldosas Cerámicas. Informe Anual (área de estudios y asuntos económicos). Castellón, 2003.

ASTER, F. Gestión de la Calidad y política de Márketing en las Empresas de la Comunidad Valenciana. Gráfiques BDM, Valencia, 1997.

CHIAVENATO, I. Introducción a la teoría general de la administración, (reimpresión de la $5^{\text {a }}$ edición de 1998) ed. McGraw-Hill, México, 2000.

CUA, K.; MCKONE, K.; SCHROEDER, R. G. Relationships between implementation of TQM, JIT, and TPM and manufacturing performance. Journal of Operations Management, v. 19, n. 6, p. 675-694, 2001.

DALMAU PORTA, J. I.; DE MIGUEL FERNÁNDEZ, E.; MIQUEL PERIS, S. Análisis estratégico de los sectores industriales y del turismo en la Comunidad Valenciana. Universidad Politécnica de Valencia, Valencia, 1993.

FULLERTON, R. R.; MCWATTERS, C. S. The production performance benefits from JIT implementation. Journal of Operations Management, v. 19, n. 1, p. 81-96, 2001.

GIFFI, C.; ROTH, A.; SEAL, G. Competing in world-class manufacturing. Irwin, Homewood, 1990.

GUNASEKARAN, A.; FORKER, L.; KOBU, B. Improving operations performance in a small company: a case study.
International Journal of Operations and Production Management, v. 20, n. 3, p. 316, 2000.

GUNN, T. H. $2^{\text {st }}$ century manufacturing: creating winning business performance, OMNEO, Essex, 1992.

KOTHA, S.; SWAMIDASS, P. M. Strategy, advanced manufacturing technology and performance: empirical evidence from U.S. manufacturing firms. Journal of Operations Management, v. 18, n. 3, p. 257-277, 2000.

LEE, K. S.; PALMER, E. An empirical examination of ISO 9000-registered companies in New Zealand. Total Quality Management, v. 10, n. 6, p. 887, 1999.

LOWE, J.; DELBRIDGE, R.; OLIVER, N. High-Performance Manufacturing - Evidence from the Automotive Components Industry. Organization Studies, v. 18, n. 5, p. 783-798, 1997.

MARÍN, F.; DELGADO, J. Las técnicas justo a tiempo y su repercusión en los sistemas de producción. Economía industrial, n. 331, p. 35-41, 2000.

MARTíNEZ SÁNCHEZ, A.; PÉREZ PÉREZ, M. Lean indicators and manufacturing strategies. International Journal of Operations and Production Management, v. 21, n. 11, p. 1433-1451, 2001.

MASKELL, B. Sistemas de datos de industrias de primer nivel mundial, TGP-Hoshin, Madrid, 1995.

NEELY, A.; RICHARDS, H.; MILLS, J.; PLATTS, K.; BOURNE, M. Designing performance measures: a structured approach. International Journal of Operations and Production Management, v. 17, n. 11, p. 1131, 1997.

ROWLEY, C. Flexible specialisation: some comparative dimensions and evidence from the ceramic tile industry. 
New Technology, Work and Employment, v. 11, n. 2, p. 125-136, 1996.

SHAH, R.; WARD, P. T. Lean manufacturing: context, practice bundles, and performance, Journal of Operations Management, v. 21, n. 2, p. 129-149, 2003.

SUZAKI, K. Competitividad en fabricacion: técnicas para la mejora continua. $3^{\mathrm{a}} \mathrm{ed}$., TGP (Tecnologías de Gerencia y Producción), Madrid, 2000.

TOMÁS CARPI, J. A.; BANYULS I LLOPIS, J.; CANO CANO, E.; CONTRERAS NAVARRO, J. L.; GALLEGO BONO, J. R.; PICHER I CAMPOS, J. V.; SUCH JUAN, J.; TORREJÓN VELARDIEZ, M. Cambio ténico-organizativo e impacto en el mercado de trabajo: La industria valenciana. Análisis de los sectores cerámico, calzado, textil y mueble. Informe de investigación. Grupo de Estudios sobre la Dinámica Industrial y Laboral (GREDIL), Universidad de Valencia, Valencia, 1996.

WHITE, R. E.; PEARSON, J. N.; WILSON, J. R. JIT manufacturing: A survey of implementations in small and large U.S. manufacturers, Management Science, v. 45, n. 1, p. 1-16, 1999.

WOMACK, J. P.; JONES, D. T.; ROOS, D. La máquina que cambió el mundo, 1 ed., McGraw-Hill Española, Madrid, 1992.

YBARRA, J. A.; GINER, J. M.; SANTA MARIA, M. J. Una política industrial para la PYME. La experiencia de la cerámica española, Economía industrial, n. 308, p. 175-185, 1996.

\section{EFFECT OF THE TYPE OF PRODUCT MANUFACTURED AND OF COMPANY SIZE ON THE PRODUCTIVE RESULTS OF CERAMIC TILE MANUFACTURERS OF THE VALENCIANA COMMUNITY}

\section{Abstract}

This article presents an analysis of data compiled from interviews with 101 production managers and from subsequent plant visits, revealing the relevance of the type of products companies manufacture and the number of employees they have in explaining the differences in their productive results. Some indicators, such as manufacturing lead times, finished product inventories and productivity per worker, differ significantly from one company to another. As a result, the parameter of production manager satisfaction varies only very slightly when grouping companies in terms of product manufactured.

Keywords: ceramic tile manufacturers, Valenciana Community, productive results. 